\title{
How Digital Health Technology Aids Physicians
}

\author{
Nik Tehrani, PhD \\ Argosy University, CA, USA
}

\begin{abstract}
There is so much health and medical information available today that physicians cannot be expected to know it all. Thus, advances in technology have become a necessity for doctors to track patient information and care, and add to patient databases for reference and to conduct research. It is important to understand the new language of digital health, such as Personal Health Record (PHR), Electronic Medical Record (EMR) and Electronic Health Record (EHR), all of which sound similar, but are not interchangeable. The ideal comprehensive IT system would empower patients, advance healthcare delivery and transform patient data into life-saving research (Kaiser, 2015). OmniFluent Health is language translation software that will allow for better patient/ practitioner communication and avoid errors. Digital technology employs the use of big data that is shared, accessed, compiled and applied using analytics. However, information transfer, especially as mandated by current ethics of use of technology, has resulted into breach of patient privacy. Improved digital technology is providing the health care field with upgrades that are necessary, electronic files and health records, from mobile apps, and remote monitoring devices.
\end{abstract}

Keywords: Personal Health Record (PHR); Electronic Health Record (EHR); digital health; remote monitoring.

The embracing of health information technology by physicians has increased, which has resulted in better patient care. But with so much new medical oriented technology, it is important to understand of the language of digital health. Personal Health Record (PHR), Electronic Medical Record (EMR) and Electronic Health Record (EHR) sound quite similar, but they are not identical and are not interchangeable. Each has its own function separate from the other. An estimated 70 percent of U.S. doctors already utilize some aspect of EHR, however, only about one quarter of those subscribe to a sophisticated multifunctional system. Ideally, a comprehensive IT system should empower patients, advance healthcare delivery and transform patient data into lifesaving research [1]. The new technological approaches to healthcare delivery have resulted in faster and more accurate diagnostic and monitoring, more sophisticated coordination across regions and agencies and sophisticated risk-checking procedures.

A patient's Personal Health Record (PHR) is a secure portal by which he/she can access the EMR to make and change appointments, check lab results, order prescriptions and modify personal information. The EMR is the electronic version of a patient's medical record that connects doctors and other caregivers with patient data from every point of

*Corresponding author: Nik Tehrani, PhD. Argosy University, CA, USA E-mail:nik@,niktehrani.com healthcare, such as x-rays, prescriptions and MRI's. The EHR is a secure electronic database information from all of a patient's EMRs under one umbrella to avoid redundant testing and errors in prescription medication [1], and also allows doctors to create a large patient database from which they can conduct research. The EMR enables doctors to electronically exchange key clinical information with other caregivers to get the broader picture of a patient's medical history [1].

Digital technologies enable analysis of patient data to present better and quicker treatment and diagnoses. E. Lee [2] points out that Dr. Watson, a computer developed by IBM, helps medical practitioners formulate more accurate diagnoses and recommend treatment. Dr. Watson helps doctors monitor the history of their patients, refer to the latest medical studies and analyze up-to-date treatment alternatives, enhancing doctors' abilities to diagnose and monitor patient health using current information [3].

According to V. Kaptelinin [4] translation technologies are making doctors more effective because they can communicate with their patients more easily by overcoming language barriers. OmniFluent Health, a product from Science Applications International Corporation (SAIC), is translation software for all medical practitioners. The software includes a mobile application (app) which allows a practitioner to ask, for example, if a patient is allergic to a certain drug. The app translates to a language that is the patient understands, lessening the possibility of prescribing the wrong 
medication. R. Symthe [5] points out that, in the United States, approximately forty seven million residents do not speak English fluently. Hence, clarifying the communication between patients and their doctors more easily will increase the doctor's patient knowledge and avoid errors.

E. Topol [6] indicates that digital technologies will allow medical practitioners to easily link up with each other without meeting face-to-face. Nearly one-third of healthcare professionals use exclusive healthcare mainstream social media networks where they can collaborate with colleagues and share resources online [7]. One social network tailored for physicians is Doximity. According to E. Lee [2], this platform allows doctors in the United States to collaborate online and discuss difficult cases. Doximity has 250,000 members, representing approximately 40 percent of all U.S. doctors who exchange information [7]. Most of the "HIPAAcompliant one-to-one messages and discussion forums focus on business challenges or diagnoses" [7].

G. Eyesenbach [3] maintains that medical digital technology, Sherpaa, helps patients and doctors connect via the phone or online, avoiding a trip to the emergency room. Reliable advice is rapidly provided to patients from medical specialists. V. Kaptelinin [4] points out that digital technology employs the use of big data that is shared, accessed, compiled and applied using analytics. The medical setting benefits by having a robust and efficient clinical and business decisionmaking platform. For instance, medical practitioners can leverage huge amounts of patient information collected from a number of sources to establish the clinical validity of particular managements and how to improve them [5]. In addition, doctors can access and share patient medical records to eliminate unnecessary medication and/or testing.

The doctor/patient experience is enhanced by technology, due to ease in cooperating with experts and physicians in innovative ways and utilizing computers that analyze patients' medical information to provide more efficient and better treatment for the patients [2]. As digital technology continues to expand the scope of medical interactions and medicine, a new revolution in the health care setting is on the horizon, such as remote health monitoring for elderly through interactive television [8] and other types of hand-held remote monitoring devices.

Digital tools have, however, been found to be incompatible with changing patient needs. Ideally, the current area of research in molecular technology, including laser guided surgeries, has fallen short of the necessary transmission of information needed to help in completing diagnostics. However, innovative diagnostics which involve extreme reliance of technology have in some instances led to misdiagnosis, leading to fatalities [4]. Further, information transfer, especially as mandated by current ethics of use of technology, has resulted into breach of patient privacy.

Improved digital technology is providing the health care field with upgrades that are necessary, electronic files and health records, from mobile apps, remote monitoring devices, and medical translation tools which help individuals to have healthier lives. All of the advancements in digital technologies are increasingly more sophisticated and are becoming a necessity. Doctors cannot avoid the rush of new advancements. And why should they? There is so much information available that physicians cannot be expected to know everything (Smythe, 2015). Digital technologies are there to help doctors work smarter and more efficiently; hence digital technology has become essential in the healthcare context.

\section{References}

1. Kaiser Permanente. (2015). Papers to pixels. Understanding the language of digital health. Retrieved from https://businessresources.kaiserpermanente.org/Global/ FileLib/kp-toolkits/Kaiser_Permanente-_EMR_infographic. pdf.

2. Lee E. 5 ways Technology is Transforming Health Care. Forbes, 2013; 15 (4):9-10

3. Eysenbach G. What is e-health? J Med Internet Res 2005; 3(2):20.

4. Kaptelinin V. Activity theory: implications for humancomputer interaction. Cambridge, MA: MIT Press; 2008:103116.

5. Smythe R. Is technology dumbing down your doctor? Forbes, 2015; 75(2): 8-10.

6. Topol E. The creative destruction of medicine: how the digital revolution will create better health care. New York: Basic Books; 2014:63-98.

7. Diana A. (2014). Information week healthcare. Healthcare social networks: New choices for doctors, patients. Retrieved from http://www.informationweek.com/healthcare/patienttools/healthcare-social-networks-new-choices-for-doctorspatients/d/d-id/1234884.

8. Spinsante S, Gambi E. Remote health monitoring for elderly through interactive television. Biomedical Engineering Online 2012; 11, 54. doi: http://dx.doi.org/10.1186/1475925X-11-54. 